\title{
Máxima Fase Estável de Lactato \\ Sanguíneo e Potência Crítica em \\ Ciclistas Bem Treinados
}

\section{Maximal Lactate Steady State and Critical Power in Well-trained Cyclists}

Renato Aparecido Corrêa Caritá Camila Coelho Greco

Benedito Sérgio Denadai

Laboratório de Avaliação da Performance Humana, Unesp Rio Claro, SP.

\section{Endereço para correspondência:} Benedito Sérgio Denadai Laboratório de Avaliação da Performance Humana - Instituto de Biociências

Avenida 24A, 1.515 - Bela Vista 13506-900 - Rio Claro, SP - Brasil

Submetido em: 27/11/2008 Versão final recebida em: 27/04/2009 Aceito em: 11/05/2009

\section{RESUMO}

O principal objetivo deste estudo foi comparar a intensidade correspondente à máxima fase estável de lactato (MLSS) e a potência crítica (PC) durante o ciclismo em indivíduos bem treinados. Seis ciclistas do sexo masculino (25,5 $\pm 4,4$ anos, 68,8 $\pm 3,0 \mathrm{~kg}, 173,0 \pm 4,0 \mathrm{~cm})$ realizaram em diferentes dias os seguintes testes: exercício incremental até a exaustão para a determinação do pico de consumo de oxigênio $\left(\mathrm{VO}_{2}\right.$ pico) e sua respectiva intensidade $\left(\mathrm{IVO}_{2}\right.$ pico); cinco a sete testes de carga constante para a determinação da MLSS e da PC; e um exercício até a exaustão na PC. A MLSS foi considerada com a maior intensidade de exercício onde a concentração de lactato não aumentou mais do que $1 \mathrm{mM}$ entre o 10 e o 30 min de exercício. Os valores individuais de potência $\left(95,100\right.$ e $110 \% \mathrm{IVO}_{2}$ pico) e seu respectivo tempo máximo de exercício (Tlim) foram ajustados a partir do modelo hiperbólico de dois parâmetros para a determinação da PC. Embora altamente correlacionadas $(r=0,99 ; p=0,0001)$, a PC $(313,5 \pm 32,3 \mathrm{~W})$ foi significantemente maior do que a MLLS $(287,0 \pm 37,8 \mathrm{~W})(p=0,0002)$. A diferença percentual da PC em relação à MLSS foi de $9,5 \pm 3,1 \%$. No exercício realizado na PC, embora tenha existido componente lento do $\mathrm{VO}_{2}\left(\mathrm{CL}=400,8 \pm 267,0 \mathrm{ml} \cdot \mathrm{min}^{-1}\right)$, o VO $\mathrm{O}_{2}$ pico não foi alcançado $(91,1 \pm 3,3 \%)$. Com base nesses resultados pode-se concluir que a PC e a MLSS identificam diferentes intensidades de exercício, mesmo em atletas com elevada aptidão aeróbia. Entretanto, o percentual da diferença entre a MLLS e PC (9\%) indica que relação entre esses dois índices pode depender da aptidão aeróbia. Durante o exercício realizado até a exaustão na $\mathrm{PC}, \mathrm{O} \mathrm{CL}$ que é desenvolvido não permite que o $\mathrm{VO}_{2}$ pico seja alcançado.

Palavras-chave: ciclismo, consumo de oxigênio, exercício aeróbio.

\section{ABSTRACT}

The main objective of this study was to compare the intensity corresponding to the maximal lactate steady state (MLSS) and critical power (CP) in well-trained cyclists. Six male cyclists ( $25.5 \pm 4.4$ years, 68.8 $\pm 3.0 \mathrm{~kg}, 173.0 \pm 4.0 \mathrm{~cm}$ ) performed in different days the following tests: incremental exercise test until exhaustion to determine peak oxygen uptake $\left(\mathrm{VO}_{2}\right.$ peak) and its respective intensity $\left(\mathrm{IVO}_{2}\right.$ peak); five to seven constant workload tests to determine MLSS and CP and; one exhaustion test at CP. MLSS was defined as the highest workload at which blood lactate concentration did not increase by more than $1 \mathrm{mM}$ between minutes 10 and 30 of the constant workload. Individual values for power-Tlim from the constant workload tests $\left(95,100\right.$ and $110 \% \mathrm{IVO}_{2}$ peak) were fit to the hyperbolic model of two-parameter to determine CP. Although highly correlated $(r=0.99 ; p=0.0001), C P(313.5 \pm 32.3 \mathrm{~W})$ was statistically higher than MLSS $(287.0 \pm 37.8 \mathrm{~W})(p=0.0002)$. The percentual difference between CP and MLSS was $9.5 \pm 3.1 \%$. During exercise performed at $\mathrm{CP}$, although a slow component of $\mathrm{VO}_{2}$ has developed $(\mathrm{SC}=$ $\left.400.8 \pm 267.0 \mathrm{ml} \cdot \mathrm{min}^{-1}\right)$, the $\mathrm{VO}_{2}$ peak was not attained $(91.1 \pm 3.3 \%$ ). Based on these results, it can be concluded that CP and MLSS identify different exercise intensities, even in athletes with high fitness level. However, the percentual difference between CP and MLSS (9\%) indicates that the relationship between these indexes may depend on aerobic fitness. During exercise performed at $\mathrm{CP}$, the $\mathrm{SC}$ does not allow $\mathrm{VO}_{2 \text { peak }}$ to be attained.

Keywords: cycling, oxygen uptake, aerobic exercise. 


\section{INTRODUÇÃO}

Durante o ciclismo estacionário de alta intensidade, a relação entre a potência externa gerada $(P)$ e o seu respectivo tempo máximo de exercício (Tlim) tem sido frequentemente descrita por um modelo

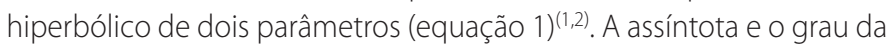
curvatura dessa relação têm sido identificados como "potência crítica" (PC) e "capacidade de trabalho anaeróbio" (CTAn), respectivamente.

$$
T \lim =\frac{C T A n}{(P-P C)}
$$

As naturezas aeróbia e anaeróbia da PC e CTAn, respectivamente, têm sido exaustivamente confirmadas por diferentes delineamentos experimentais (2-4). Uma importante implicação da determinação da PC, tanto para a prescrição do exercício aeróbio bem como na elaboração de delineamentos experimentais, é a identificação do limite entre o domínio pesado e severo de exercício. Por definição, durante o exercício realizado no domínio pesado, a concentração de lactato sanguíneo ([La]) e o consumo de oxigênio $\left(\mathrm{VO}_{2}\right)$ apresentarão estabilidade, embora este último possa apresentar um valor maior que o predito pela relação $\mathrm{VO}_{2}$ vs. carga, pelo surgimento do componente lento (CL). No domínio severo (ainda em intensidades submáximas), a [La] não apresenta estabilidade e o pico do consumo máximo $\left(\mathrm{VO}_{2}\right.$ pico é obtido quando o exercício é realizado até a exaustão(1).

Alternativamente, Pringle e Jones (2) sustentam que a intensidade correspondente à máxima fase estável de lactato (MLSS), e não à PC, seria o limite entre o domínio pesado e severo, já que a [La] e o $\mathrm{VO}_{2}$ não apresentaram estabilidade acima da MLSS. Entretanto, os dois únicos estudos realizados no ciclismo estacionário(2,4) verificaram que a PC é significantemente correlacionada, porém maior que a MLSS. Desse modo, a PC e a MLSS representariam diferentes intensidades de exercício e consequentemente apresentam respostas agudas ao esforço ( $[\mathrm{L} a], \mathrm{VO}_{2}$ e tempo de exaustão) também distintas e potencialmente não podem ser utilizadas de modo intercambiável.

Deve-se salientar, entretanto, que Dekerle et al. ${ }^{(4)}$ analisaram a relação entre MLSS de PC em indivíduos ativos $\left(\mathrm{VO}_{2}\right.$ pico $\left.=50,8 \mathrm{ml} \cdot \mathrm{min}^{-1} \mathrm{~kg}^{-1}\right)$, enquanto Pringle e Jones ${ }^{(2)}$ analisaram indivíduos com diferentes níveis de aptidão aeróbia (dois ativos, um corredor, dois duatletas e três ciclistas; $\mathrm{VO}_{2}$ pico $\left.=51,7 \mathrm{ml} \cdot \mathrm{min}^{-1} \mathrm{~kg}^{-1}\right)$. Para o nosso conhecimento, nenhum estudo analisou a relação entre MLSS e PC em indivíduos com elevada aptidão aeróbia no ciclismo. Esse aspecto pode ser particularmente importante, pois encontramos recentemente em nosso laboratório ${ }^{(5)}$ que a relação entre a PC e um índice associado à resposta de lactato ao exercício (i.é, limiar de lactato - LL) durante o ciclismo pode ser modificada pela aptidão aeróbia. Neste estudo verificamos que, em indivíduos bem treinados no ciclismo, a PC se localiza em um percentual maior da diferença entre o $\mathrm{LL}$ e o $\mathrm{VO}_{2}$ pico, sugerindo que o treinamento aeróbio pode modificar mais a resposta de lactato ao exercício do que a PC. Baseada nesse aspecto, a hipótese central deste estudo é que a intensidade correspondente à PC seja similar à MLSS em ciclistas bem treinados. Portanto, o principal objetivo deste estudo foi comparar a MLSS e a PC durante o ciclismo em indivíduos bem treinados. Secundariamente, foi objetivo também descrever as repostas metabólicas durante o exercício realizado na PC até a exaustão voluntária nesses atletas.

\section{MÉTODOS}

\section{Sujeitos}

Participaram do estudo seis ciclistas do sexo masculino (25,5 $\pm 4,4$ anos, 68,8 $\pm 3,0 \mathrm{~kg}, 173,0 \pm 4,0 \mathrm{~cm}$ ), com pelo menos cinco anos de experiência na modalidade. Os atletas treinavam de seis a sete vezes por semana (volume $=430 \pm 40 \mathrm{~km}$ ) e participavam de competições em nível estadual e nacional. Os indivíduos foram orientados a não realizar treinamento intenso e não ingerir bebidas contendo cafeína e álcool nas 24 horas que antecederam as sessões experimentais. Este estudo foi aprovado pelo Comitê de Ética em Pesquisa da Universidade Estadual Paulista - Unesp - Campus Rio Claro (Processo CEP 144/2007).

\section{Delineamento experimental}

Os atletas compareceram ao laboratório entre sete e nove vezes dentro de um período de três semanas. Inicialmente os atletas realizaram um teste incremental para determinar o limiar anaeróbio (LAn), o $\mathrm{VO}_{2}$ pico e sua respectiva intensidade $\left(\mathrm{IVO}_{2}\right.$ pico). Durante as demais sessões, os atletas realizaram exercícios de carga constante para determinar a MLSS e a PC. Cada um desses testes de carga constante foi realizado em ordem aleatória. Os testes foram separados por pelo menos $48 \mathrm{~h}$ e foram realizados nos mesmos horários do dia ( $\pm 2 \mathrm{~h}$ ) em um laboratório com temperatura controlada $\left(21-22^{\circ} \mathrm{C}\right)$.

\section{Protocolo incremental}

Os sujeitos foram submetidos a um exercício incremental em cicloergômetro de frenagem eletromagnética (Excalibur Sport, Lode BV Croningen, Holanda) com frequência de pedalada entre 70 e 90rpm. A intensidade inicial foi de $100 \mathrm{~W}$ com incrementos de $25 \mathrm{~W}$ a cada três minutos até a exaustão voluntária. Ao final de cada estágio houve coleta de sangue do lóbulo da orelha para a dosagem do lactato sanguíneo (YSL 2300 STAT, Yellow Springs, Ohio, EUA). O VO ${ }_{2}$ foi mensurado respiração a respiração durante todo o protocolo a partir do gás expirado (Quark PFTergo - Cosmed, Itália), sendo os dados reduzidos às médias de 15 s. A calibração dos sistemas de análise das concentrações de $\mathrm{O}_{2}$ e $\mathrm{CO}_{2}$ foi feita antes de cada teste, usando ar ambiente e um gás com concentrações conhecidas de $\mathrm{O}_{2}$ e $\mathrm{CO}_{2}$ de acordo com as instruções do fabricante. A turbina do analisador foi calibrada através de uma seringa de três litros. $\mathrm{OVO}_{2}$ pico foi considerado como o maior valor de 15 s obtido durante o teste. A IVO 2 pico foi considerada como sendo a menor intensidade de exercício na qual ocorreu o $\mathrm{VO}_{2}$ pico. O LAn foi encontrado através de uma interpolação linear (lactato vs. intensidade), considerando uma concentração fixa de lactato de $3,5 \mathrm{mM}^{(6)}$.

\section{Protocolo de carga constante para a determinação da MLSS}

Foram realizados de dois a quatro testes de carga constante com duração de 30 minutos em diferentes intensidades para a determinação da potência, [La] e frequência cardíaca (FC) correspondentes à MLSS. A primeira carga foi equivalente a 100\% do LAn. Dependendo do comportamento da lactato sanguíneo, foram realizados outros testes de carga constante abaixo ou acima dessa intensidade. No $10^{\circ}$ e $30^{\circ}$ minuto dos testes foram coletadas amostras de sangue do lóbulo da orelha para a determinação da [La]. A MLSS foi considerada como sendo a mais alta carga na qual foi observado aumento menor ou igual a 1,0mM entre o $10^{\circ}$ e $30^{\circ}$ minuto de exercício, conforme proposto por Beneke ${ }^{(7)}$.

\section{Protocolo de carga constante para a determinação da PC}

Inicialmente os indivíduos realizaram um aquecimento de $10 \mathrm{mi}$ nutos a $60 \%$ da $\mathrm{IVO}_{2}$ pico. A seguir, repousaram por cinco minutos no cicloergômetro e logo em seguida a intensidade foi ajustada em 95\%, $100 \%$ ou $110 \%$ da $\mathrm{IVO}_{2}$ pico até a exaustão voluntária ou até que o indivíduo não pudesse manter a intensidade estipulada (cadência < 67rpm). OTlim foi considerado como o tempo total de esforço mantido no respectivo $\% \mathrm{IVO}_{2}$ pico e expresso em segundos. Os valores individuais de potência e Tlim obtidos durante os testes de carga constante foram ajustados a partir do modelo hiperbólico de dois parâmetros (equação 1). 


\section{Protocolo de carga constante na intensidade correspon- dente à $\mathrm{PC}$}

Inicialmente os indivíduos realizaram um aquecimento de $10 \mathrm{mi}$ nutos a $60 \%$ da $\mathrm{IVO}_{2}$ pico. A seguir, repousaram por cinco minutos no cicloergômetro e logo em seguida a intensidade foi ajustada em 100\% PC e mantida até a exaustão voluntária ou até que o indivíduo não pudesse manter a intensidade estipulada (cadência $<67 \mathrm{rpm}$ ). O VO e a FC foram medidos durante todo o teste. A [La] foi medida no $10^{0}$ min e na exaustão. $\mathrm{O} C \mathrm{~L}$ foi determinado como a diferença entre os valores do $\mathrm{VO}_{2}$ entre o 3 - min (média entre 2,75 ao 3,25으 minuto) e o valor na exaustão (média dos últimos 30s).

\section{ANÁLISE ESTATÍSTICA}

Os dados estão expressos como média \pm DP. A normalidade dos dados foi verificada através do teste de Shapiro-Wilk. As intensidades correspondentes à MLSS e PC foram analisadas através do teste $t$ de Student para dados pareados e pelo teste de correlação de Pearson. Para a comparação entre a MLSS e a PC foi calculado também o tamanho do efeito (effect size - ES). O nível de significância foi mantido em $p \leq \_0,05$.

\section{RESULTADOS}

As variáveis obtidas no teste incremental estão apresentadas na tabela 1.

Tabela 1. Valores médios \pm DP das variáveis obtidas no protocolo incremental. $N=6$.

\begin{tabular}{l|c}
\hline & Média $\pm \mathrm{DP}$ \\
\hline $\mathrm{VO}_{2}$ pico $\left(\mathrm{ml}_{\mathrm{k}} \mathrm{kg}^{-1} \cdot \mathrm{min}^{-1}\right)$ & $62,7 \pm 5,6$ \\
\hline $\mathrm{IVO}_{2}$ pico $(\mathrm{W})$ & $366,5 \pm 28,5$ \\
\hline FCmax (bpm) & $189,3 \pm 10,5$ \\
\hline PLAn (W) & $284,3 \pm 25,6$ \\
\hline
\end{tabular}

$\mathrm{VO}_{2}$ pico - pico do consumo de oxigênio; $\mathrm{VO}_{2}$ pico - intensidade correspondente ao $\mathrm{VO}_{2}$ pico; $\mathrm{FCmax}$ - frequência cardíaca máxima; PLAn - potência no limiar anaeróbio.

A tabela 2 mostra os valores da potência, Tlim, FC e [Lac] correspondentes a 95, 100 e $110 \% \mathrm{NVO}_{2}$ max.

Tabela 2. Valores médios \pm DP das variáveis obtidas a 95, 100 e 110\% da intensidade correspondente ao pico do consumo de oxigênio $\left(\mathrm{IVO}_{2}\right.$ pico). $\mathrm{N}=6$.

\begin{tabular}{c|c|c|c|c}
\hline $\begin{array}{c}\text { Intensidade } \\
\left(\% \text { IVO }_{2} \text { pico) }\right.\end{array}$ & Potência (W) & Tlim (s) & FC (bpm) & [Lac] (mM) \\
\hline 95 & $348,1 \pm 27,3$ & $705,8 \pm 207,6$ & $184,6 \pm 5,0$ & $9,8 \pm 0,4$ \\
\hline 100 & $366,5 \pm 28,5$ & $484,0 \pm 128,2$ & $186,5 \pm 8,7$ & $10,7 \pm 1,4$ \\
\hline 110 & $402,8 \pm 31,3$ & $238,0 \pm 55,8$ & $185,3 \pm 9,1$ & $9,6 \pm 5,0$ \\
\hline
\end{tabular}

A figura 1 mostra os valores médios \pm DP da MLSS, da PC e a sua diferença percentual. A PC $(313,5 \pm 32,3 \mathrm{~W})$ foi significantemente maior do que a MLSS $(287,0 \pm 37,8 \mathrm{~W})(\mathrm{p}=0,0002 ; \mathrm{ES}=0,82)$. As duas variáveis foram altamente correlacionadas $(r=0,99 ; p=0,0001)$. A diferença percentual da PC em relação à MLSS foi de 9,5 $\pm 3,1 \%$.

A tabela 3 mostra os valores obtidos durante o exercício realizado até a exaustão na PC. O $\mathrm{VO}_{2}$ no 3 - minuto foi significantemente menor do que ao final do exercício, determinando um CL de 400,8 $\pm 267,0 \mathrm{ml}$. $\min ^{-1}$. Do mesmo modo, a [Lac] foi maior ao final do exercício do que no $10^{0}$ min. O Tlim na PC foi de $23,5 \pm 5,0$ min.

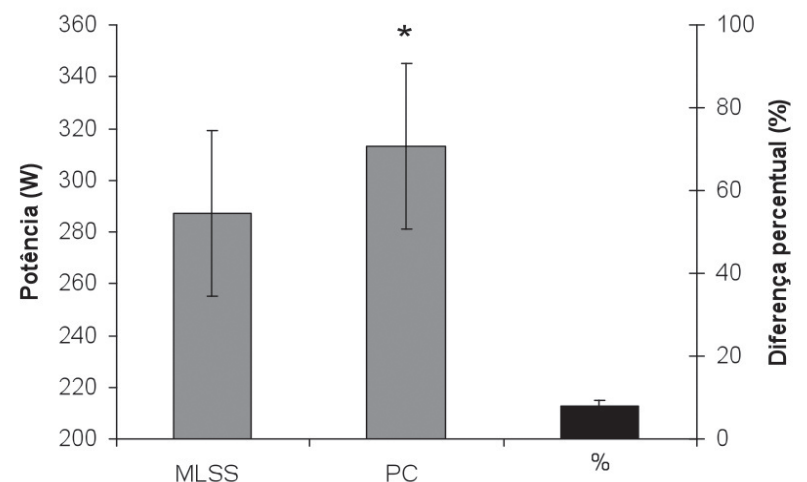

Figura 1. Valores médios \pm DP das intensidades correspondentes à máxima fase estável de lactato (MLSS) e da potência crítica (PC) e da sua respectiva diferença percentual (\%). ${ }^{*} P=0,0002$ em relação à MLSS.

Tabela 3. Valores médios \pm DP da resposta metabólica e do tempo de exaustão na intensidade correspondente à potência crítica. $\mathrm{N}=6$.

\begin{tabular}{c|c|c|c|c|c|c|c}
\hline & $\begin{array}{c}\mathrm{VO}_{2} 3 \mathrm{~min} \\
\left(\mathrm{ml}_{\mathrm{min}}^{-1}\right)\end{array}$ & $\begin{array}{c}\mathrm{VO}_{2} \text { final } \\
\left(\mathrm{ml}_{\mathrm{min}}^{-1}\right)\end{array}$ & $\% \mathrm{VO}_{2}$ pico & $\begin{array}{c}\mathrm{CL} \\
\left(\mathrm{ml}_{\mathrm{min}}^{-1}\right)\end{array}$ & $\begin{array}{c}{[\mathrm{Lac}] 10 \mathrm{~min}} \\
(\mathrm{mM})\end{array}$ & $\begin{array}{c}{[\mathrm{Lac}] \text { final }} \\
(\mathrm{mM})\end{array}$ & $\begin{array}{c}\text { Tlim } \\
(\mathrm{min})\end{array}$ \\
\hline Média & $3.481,8$ & $3.877,2^{*}$ & 91,1 & 400,8 & 6,3 & $8,4^{+}$ & 23,5 \\
\hline DP & 439,0 & 393,4 & 3,3 & 267,0 & 2,0 & 2,8 & 5,0 \\
\hline
\end{tabular}

$\mathrm{VO}_{2} 3 \mathrm{~min}$ - consumo de oxigênio no 30 minuto; $\mathrm{VO}_{2}$ final - consumo de oxigênio no final do exercício; \% $\mathrm{VO} \mathrm{O}_{2}$ pico - percentual do consumo de oxigênio pico atingido no final do exerć́cio; CL - componente lento; [Lac]10min - concentraçăo de lactato no 10ํำ minuto; [Lac]final - concentraçăo de lactato no final do exercício; Tlim - tempo de exaustão na potência crítica. ${ }^{*} \mathrm{P}<0,05$ em relaçăo ao $\mathrm{VO}_{2} 3 \mathrm{~min} ;{ }^{+} \mathrm{P}<0,05$ em relação a [La]10min.

\section{DISCUSSÃO}

O principal achado deste estudo foi que, embora significantemente correlacionadas, a PC é maior do que a MLSS em ciclistas bem treinados. Esse dado contraria, pelo menos em parte, a nossa hipótese inicial de que em indivíduos bem treinados aerobicamente, a MLSS seria similar à PC. Deve-se ressaltar, entretanto, que o percentual da diferença entre a MLSS e PC em nosso estudo (9\%) é aparentemente menor do que o encontrada por Dekerle et al.(4) em indivíduos ativos (16\%), sugerindo que a relação entre esses dois índices pode depender da aptidão aeróbia.

Um primeiro aspecto que deve ser analisado neste estudo é a validade do protocolo utilizado para determinar a PC, já que este índice é protocolo-dependente ${ }^{(8,9)}$. Diversos estudos demonstraram que a PC é dependente da duração (Tlim) que as cargas selecionadas permitem (i.é., quanto maior a duração das cargas utilizadas na predição menor a PC). Nesse sentido, di Prampero(10) aponta que as cargas selecionadas para a determinação da PC deveriam permitir que o VO ${ }_{2}$ pico seja alcançado durante o exercício de carga constante, ou seja, Tlim entre dois e $15 \mathrm{~min}$. No presente estudo, o $\mathrm{VO}_{2}$ não foi medido durante os testes para determinar a PC. Entretanto, os Tlim ficaram dentro do sugerido pela literatura ( 4-12 min). Além disso, verificamos recentemente em nosso laboratório que as cargas aqui empregadas (i.é., 95, 100 e 110\% $\mathrm{IVO}_{2}$ pico) permitem que o $\mathrm{VO}_{2}$ pico seja atingido antes da exaustão, independente do nível de aptidão aeróbia(11). Como no estudo de Dekerle et al.(4) esses aspectos também foram observados, nos parece possível a comparação entre os dois trabalhos.

Os domínios de intensidade de exercício (i.é., moderado, pesado e severo) são caracterizados por diferentes respostas de pH, [La] e VO Além disso, as adaptações fisiológicas crônicas ao exercício podem ser também específicas ao domínio onde o treinamento foi preferencialmente realizado ${ }^{(12)}$. Assim, muitos estudos têm analisado a validade dos protocolos e dos índices para identificar os limites entre os domínios, particularmente entre os domínios pesado e severo. Inicialmente, tanto a MLSS como a PC foram utilizadas como os índices que determinaram 
esse limite, significando que as mesmas identificariam intensidades similares de exercício. No ciclismo, entretanto, Dekerle et al. ${ }^{(4)}$ e Pringle e Jones ${ }^{(2)}$ verificaram que a PC é significantemente maior do que a MLSS, evidenciando que esses índices representam diferentes intensidades e poderiam localizar-se em diferentes domínios de intensidade de exercício. Salienta-se que o estudo de Dekerle et al.(4) foi o único que analisou um grupo homogêneo de indivíduos quanto ao estado de treinamento (i.é., ativos). Recentemente, verificamos em nosso laboratório que durante o ciclismo as relações entre $\mathrm{LL}, \mathrm{PC}$ e $\mathrm{VO}_{2}$ pico podem ser modificadas pela aptidão aeróbia( ${ }^{(5)}$. Neste estudo, encontramos que a PC localiza-se em $75 \%$ da diferença entre o $\mathrm{LL}$ e o $\mathrm{VO}_{2}$ pico (i.é., $75 \% \Delta$ ). Por outro lado, Neder et al. ${ }^{(13)}$ verificaram em indivíduos sedentários que a PC foi equivalente a $62 \% \triangle$. Esses dados em conjunto evidenciam que o LL pode aumentar mais com o treinamento aeróbio do que a PC. Com isso, foi hipotetizado no presente estudo que a MLSS e a PC pudessem ser similares em ciclistas bem treinados. Entretanto, verificou-se que a PC foi significantemente maior do que a MLSS, não sustentando, pelo menos em parte, nossa hipótese inicial. Ressalta-se, porém, que a diferença percentual entre a MLSS e PC em nosso estudo (9\%) é aparentemente menor do que a encontrada por Dekerle et al. ${ }^{(4)}$ em indivíduos ativos (16\%), sugerindo novamente que a relação entre resposta de lactato (LL e MLSS) e PC pode depender da aptidão aeróbia.

Inicialmente, a PC foi conceituada como a intensidade de exercício que pode ser teoricamente sustentada indefinidamente ${ }^{(14)}$. Entretanto, está amplamente descrito que fatores como a depleção de substratos e os relacionados à regulação da temperatura corporal podem contribuir para a fadiga durante o exercício prolongado, sugerindo que essa expressão matemática não providencia uma intensidade que pode ser mantida indefinidamente. Mais recentemente, alguns autores têm proposto que a PC identifica a intensidade que permite um esforço prolongado, com a [La] se mantendo constante e o $\mathrm{VO}_{2}$ não atinge seus valores máximos quando o exercício é realizado até a exaustão ${ }^{(1,15)}$. 0 Tlim na PC encontrado em nosso estudo (23 min) está dentro da grande faixa de variação existente na literatura (18-60 min)(3,16-18). Essa grande variabilidade pode ser explicada por um aspecto já anteriormente discutido, ou seja, o fato de a PC ser protocolo-dependente (seleção das cargas e modelo matemático utilizado). Destaca-se que neste domínio de intensidade, pequenas variações na intensidade (5-10\%) podem permitir variações de até 100\% no Tlim. Nesse sentido, nosso Tlim (23 min) é compatível com o Tlim (55 min) encontrado na MLSS de ciclistas treinados ${ }^{(17)}$, pois a PC foi maior do que a MLSS em 9\%. Um segundo aspecto a ser ressaltado é que, similar ao obtido por Brickley et al. ${ }^{(18)} \mathrm{e}$ McLellan e Cheung ${ }^{(19)}$, durante o exercício na PC a [La] não apresentou estabilidade, contrariando o proposto por Poole et al.(1), como o citado

\section{REFERÊNCIAS BIBLIOGRÁFICAS}

1. Poole DC, Ward SA, Gardner G, Whipp BJ. Metabolic and respiratory profile of the upper limit for prolonged exercise in man. Ergonomics. 1988;31:1265-79.

2. Pringle JS, Jones AM. Maximal lactate steady state, critical power and EMG during cycling. Eur J Appl Physiol. 2002;88:214-26

3. Jenkins DG, Quigley BM. Blood lactate in trained cyclists during cycle ergometry at critical power. Eur J Appl Physiol. 1990;61:278-83.

4. Dekerle J, Baron B, Dupont L, Vanvelcenaher J, Pelayo P. Maximal lactate steady state, respiratory compensation threshold and critical power Eur J Appl Physiol. 2003;89:281-8.

5. Caputo $\mathrm{F}$, Denadai BS. Does the $75 \%$ of the difference between the $\mathrm{VO}_{2}$ at lactate threshold and $\mathrm{VO}_{2}$ max lie at severe intensity domain in well trained cyclists? Sci Sports in press.

6. Denadai BS, Figuera, TR, Favaro ORP, Gonçalves M. Effect of the aerobic capacity on the validity of the anaerobic threshold for determination of the maximal lactate steady state in cycling. Braz J Med Biol Res. 2004;37:1551-6.

7. Beneke R. Maximal lactate steady state concentration (MLSS): experimental and modelling approaches. Eur J Appl Physiol. 2003:8:361-9.

8. Bishop D, Jenkins DG, Howard A. The critical power function is dependent on the duration of the predictive exercise tests chosen. Int J Sports Med. 1998;9:125-9.

9. Calis JFF, Denadai BS. Influência das cargas selecionadas na determinação da potência crítica determinada no ergômetro de braço em dois modelos lineares. Rev Bras Med Esporte. 2000;6:1-4.

10. di Prampero PE. The concept of critical velocity: a brief analysis. Eur J Appl Physiol Occup Physiol. 1999;80:162-4. anteriormente. Em nosso estudo esse aspecto já poderia ser esperado, pois a PC foi maior do que a MLSS, que por definição representa a maior intensidade de exercício onde a [La] apresenta estabilidade. Quanto ao comportamento do $\mathrm{VO}_{2}$, tanto os valores de $\mathrm{CL}$ encontrados como o não alcance do VO 2 pico (91\%) ao final do exercício são compatíveis com os modelos teóricos de que a PC seria o limite entre os domínios pesado e severo. Ressalta-se apenas que em nosso estudo os indivíduos não se exercitaram até a exaustão acima da PC (p.ex., 5\% acima), para confirmar se $\mathrm{VVO}_{2}$ pico seria alcançado durante essa intensidade.

Verificamos recentemente em nosso laboratório ${ }^{(20)}$ que ciclistas bem treinados exercitando-se acima da MLSS (5\%), mas provavelmente abaixo da PC (se considerados os dados do presente estudo), apresentaram $\mathrm{CL}$ menor $\left(\mathrm{CL}=106 \mathrm{ml} . \mathrm{min}^{-1}\right)$ do que o reportado neste estudo $\left(400,8 \mathrm{ml} \mathrm{min}^{-1}\right)$ e que também não levou ao $\mathrm{VO}_{2}$ pico (89\%). Assim, parecem existir claramente intensidades de exercício dentro do domínio pesado (> MLSS e $\leq$ PC), onde a [La] não apresenta estabilidade, o CL é desenvolvido, mas o $\mathrm{VO}_{2}$ pico não é alcançado.

Uma possível limitação deste estudo seria o tamanho da amostra analisada. Do ponto de vista operacional, deve-se considerar a dificuldade de recrutarem-se atletas bem treinados que tenham a disponibilidade de participar de um protocolo que prevê a vinda ao laboratório por sete a nove vezes, em um período de três semanas. Uma amostra pequena poderia ter levado ao erro do tipo I (assumir que a MLSS e PC são similares quando de fato não são), comportamento este que não foi observado, pois a PC foi estatisticamente ( $p=0,0002)$ maior do que a MLSS. Além disso, o cálculo do tamanho do efeito $(E S=0,82)$ revela alta probabilidade de a PC e a MLSS identificarem diferentes intensidades de exercício.

Finalmente, para a elaboração de delineamentos experimentais em ciclistas bem treinados onde seja importante a seleção de intensidades dentro do domínio pesado e/ou severo, não é possível utilizar-se a MLSS, pois a PC parece ser o limite entre esses domínios. Do mesmo modo, a prescrição do treinamento aeróbio com base na MLSS ou PC não permite respostas agudas e provavelmente crônicas similares. Portanto, para essa finalidade, esses índices não devem ser utilizados de modo intercambiável.

\section{CONCLUSÃO}

Com base nesses resultados pode-se concluir que, embora a PC e a MLSS possam ter excelente validade para identificar a capacidade aeróbia, as mesmas identificam diferentes intensidades de exercício, mesmo em atletas com elevada aptidão aeróbia. O percentual da diferença entre a MLSS e PC (9\%) encontrado neste estudo indica que relação entre esses dois índices pode depender da aptidão aeróbia. Durante o exercício realizado até a exaustão na $\mathrm{PC}$, a [La] não apresenta estabilidade, mas o $\mathrm{CL}$ que é desenvolvido não permite que o $\mathrm{VO}_{2}$ pico seja alcançado.
11. Caputo F, Denadai BS. The highest intensity and the shortest duration permitting attainment of maximal oxygen uptake during cycling: effects of different methods and aerobic fitness level. Eur Appl Physiol. 2008;103:47-7.

12. Greco CC, Caputo F, Denadai BS. Critical power and maximal oxygen uptake: Estimating the upper limit of the severe domain, a new challenge? Sci Sports. 2008;23:216-22.

13. Neder JA, Jones PW, Nery LE, Whipp BJ. The effect of age on the power/duration relationship and the intensity-domain limits in sedentary men. Eur J Appl Physiol. 2000;82:326-32.

14. Monod H, Scherrer J. The work capacity of a synergic muscular group. Ergonomics. 1965;8:329-38

15. Gaesser GA, Wilson LA. Effects of continuous and interval training on the parameters of the power-endurance relationship for high intensity exercise. Int J Sports Med. 1988;9:417-21.

16. Housh DJ, Housh TJ, Bauge SM. The accuracy of the critical power test for predicting time to exhaustion during cycle ergometer. Ergonomics. 1989;32:997-1004

17. Baron B, Noakes TD, Dekerle J, Moullan F, Robin S, Matran R, Pelayo P. Why does exercise terminate at the maximal lactate steady state intensity? Br J Sports Med. 2008;42:528-33.

18. Brickley G, Doust J, Williams CA. Physiological responses during exercise to exhaustion at critical power. Eur J Appl Physiol. 2002;88:146-51

19. McLellan TM, Cheung KS. A comparative evaluation of the individual anaerobic threshold and the critical power. Med Sci Sports Exerc. 1992;24:543-50.

20. Barbosa LF, de Souza MR, Pelarigo JG, Caritá RAC, Caputo F, Denadai BS, Greco CC Máxima fase estável de lactato sanguíneo e o limite superior do domínio pesado em ciclistas treinados. Rev Bras Cin Desemp Human 2009;11:320-5. 